of osteoarthritis (OA). Studies to evaluate whether dietary or supplementary antioxidants are beneficial to patients with OA have shown contradictory results. This study by Wang et al. is the first to assess the effects of antioxidants on the knee cartilage and bone of healthy middle-aged individuals before any onset of $O A$.

A cohort of 293 healthy individuals $(63 \%$ female, mean age 58 years) completed a food frequency questionnaire and underwent MRI for analysis of tibial cartilage volume and tibial plateau cross-sectional area (for tibial bone size), as well as for the detection of cartilage defects and bone-marrow lesions.

Increase in bone size is associated with onset and progression of OA; bone-marrow lesions are associated with pain and progressive joint-space loss in OA. Vitamin C and fruit intake were found to be inversely proportional to bone size and the presence of bone lesions in the healthy study participants; however, vegetable intake was not. Lutein and zeaxanthin intake was associated with a decreased risk of cartilage defects, independent of vitamin C intake. Betacryptoxanthin intake was associated with decreased bone size, independent of vitamin $E$ intake. Vitamin $E$ intake was associated with increased bone size, independent of vitamin $\mathrm{C}$ intake, and hence might exert negative effects on bone.

The authors conclude that consumption of fruit, an important source of vitamin $\mathrm{C}$, might be beneficial for bone health; however, they do note that larger longitudinal studies should be conducted to confirm this finding.

Original article Wang Y et al. (2007) Effect of antioxidants on knee cartilage and bone in healthy, middleaged subjects: a cross-sectional study. Arthritis Res Ther 9: R66

\section{TNF antagonists decrease excess mortality in RA patients}

Patients with rheumatoid arthritis $(R A)$ are at increased risk of death from cardiovascular events, cancer and infections. Tumor necrosis factor (TNF) antagonists, used to treat severe $\mathrm{RA}$, have been associated with adverse cardiac events, serious infections and malignancies. Carmona et al., therefore, investigated the effect of TNF antagonists on mortality in patients with RA. The authors analyzed outcomes in two Spanish cohorts: BIOBADASER, a registry to follow up RA patients treated with biological agents, and EMECAR, a registry of patients not treated with TNF antagonists. There were 789 patients in each cohort, and follow-up was 5 years. Death rates were compared with the general Spanish population rate.

There were 75 deaths in the EMECAR cohort, compared with 50 expected, and 20 deaths in the BIOBADASER cohort, compared with 38 expected. Incidence of cardiovascular events and cancer was significantly higher in EMECAR than BIOBADASER (relative risk [RR] 6.3 for ischemic heart disease, 7.1 for cardiac failure, 5.9 for stroke, and 2.9 for cancer), whereas the latter cohort had a higher rate (RR 1.6) of serious infections. The mortality RR for BIOBADASER versus EMECAR was 0.32 for all-cause mortality, 0.58 for cardiovascular deaths, 0.52 for deaths from infection and 0.36 for cancer deaths.

The authors conclude that mortality in RA patients treated with anti-TNF agents is not greater than that in the general population, and is lower than the rate in RA patients not treated with TNF antagonists.

Original article Carmona L et al. (2007) All-cause and cause-specific mortality in rheumatoid arthritis are not greater than expected when treated with tumour necrosis factor antagonists. Ann Rheum Dis 66: 880-885 